Tomás Fernández. "Los dos polos de la biografía antigua y la hagiografía".

Circe, de clásicos y modernos 24/2 (julio-diciembre 2020).

DOI: http://dx.doi.org/10.19137/circe-2020-240203

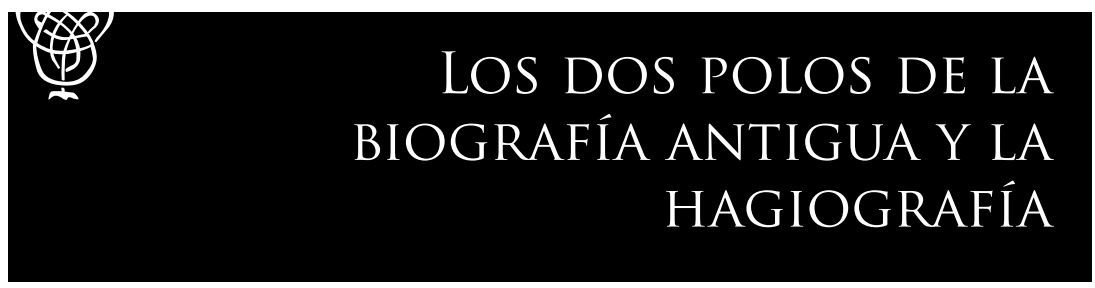

\author{
Tomás Fernández [Conicet - Universidad de Buenos Aires] \\ [Tomas.Fernandez@ conicet.gov.ar] \\ ORCID: 0000-0001-8730-0249
}

Resumen: La biografía griega puede asociarse a dos polos principales: en uno predomina el eje sintagmático, con sucesión, y en el otro el paradigmático, sin sucesión. El primero se relaciona con la historiografía, tiene un método cercano a la investigación o iơooí $\alpha$ y tiene su tipo ideal más difundido en la biografía energética o peripatética (Plutarco); el segundo se liga a la retórica demostrativa, su método es próximo al del anticuarianismo y aparece en la biografía alejandrina (Suetonio). Esta contribución sugiere que en la hagiografía típica esta oposición se verifica en un tratamiento relativamente separado de "vida" y "conducta" - sintetizada

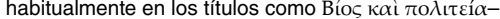
la sucesión de hechos situados en el tiempo en los que se manifiesta un carácter más o menos intemporal. Para ello, discute las características principales de la biografía; expone el sistema de F. Leo sobre la biografía clásica; se detiene en el cronotopo de M. Bajtín sobre biografía y autobiografía antigua, y en su deuda con autores como Leo o G. Misch; expone ciertas características del discurso hagiográfico y de su particular cronotopo; ofrece una breve conclusión.

Palabras clave: biografía - hagiografía - Bajtín - Leo - géneros.

\section{The two poles of ancient biography and hagiography}

Abstract: Greek biography can be associated to two main poles: on one of them the syntagmatic axis, with succession, predominates; on the other, the paradigmatic axis, without succession. The first pole is related to historiography and has a method closer to i $\sigma \tau \tau$ coí $\alpha$; its best-known ideal type is the energetic or Peripatetic hagiography (Plutarch). The second pole is related to demonstrative rhetoric, its method is closer to antiquarianism and it appears in the analytic or Alexandrian biography (Suetonius). This contribution will suggest that in the usual hagiography this opposition is visible in a separated treatment of "life" and "deeds", often sum-

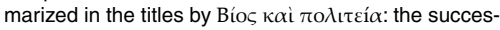
sion of facts, situated in time and space, as opposed to a timeless character, which reveals itself rather than becomes. To this aim, I will discuss the basic features of biography; describe F. Leo's theory about classical biography; examine M. Bakhtin's biographical chronotope, and his indebtedness with authors such as Leo or G. Misch; analyse some crucial features of hagiographical discourse and of its peculiar chronotope; and offer a brief conclusion.

Keywords: biography - hagiography - Bakhtin - Leo genres 


\section{La unidad, lo universal, lo sensible}

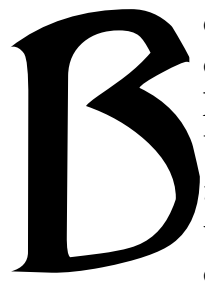

orges realizó dos grandes declaraciones sobre los relatos biográficos. Una figura en su Evaristo Carriego: "Que un individuo quiera despertar en otro individuo recuerdos que no pertenecieron más que a un tercero es una paradoja evidente"1. Esa paradoja, que también se aplica a la hagiografía, tal vez se funde en una imposibilidad compartida con la historia y con cualquier género mimético de lo real. El segundo juicio de Borges aparece en la "Biografía de Tadeo Isidoro Cruz": "Cualquier destino, por largo y complicado que sea, consta en realidad de un solo momento: el momento en que el hombre sabe para siempre quién es"2. Esta frase, tan apropiada en la narración de la vida de un gaucho argentino como Cruz, el gran compañero de Martín Fierro, tiene, en realidad, antecedentes clásicos que se remontan, por lo menos, al concepto aristotélico de $\dot{\varepsilon} v \tau \varepsilon \lambda \hat{\varepsilon} \chi \varepsilon\llcorner\alpha$, entendida como realidad completa o

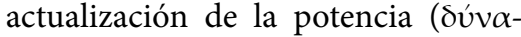
$\mu \iota \varsigma)$. La importancia de este concepto en la biografía antigua fue señalada, entre otros, por BAjTín. Según este autor, la esencia del hombre antiguo

1 Borges (1974: 113). Para desarticular esta paradoja, Borges burló las expectativas genéricas de sus lectores, presentando como una biografía algo que, en realidad, era una historia de Palermo.

2 Borges (1974: 158). es su figura pública. Por ende, tras hablar de San Agustín y de la autobiografía, se centra en las formas de la autoconciencia pública del hombre ${ }^{3}$. Analiza a continuación las formas biográficas que llegan a la madurez en el período helenístico y romano: "En primera instancia, notemos la influencia de Aristóteles sobre los métodos caracterológicos de los biógrafos antiguos, y más precisamente la doctrina de la entelequia como fin último y al mismo tiempo causa primera de la evolución"4. Bajtín remitía inmediatamente antes a la cuestión de la autoconciencia; esta autoconciencia, combinada con el problema de la entelequia espeja, de manera muy precisa, la frase de Borges acerca de que un destino, aunque sea largo $\mathrm{y}$ aventurero, puede resumirse en el instante en que el individuo, en términos de Borges, "sabe para siempre quién es" o, en términos bajtinianos, "alcanza la autoconciencia" de su potencial realizado (que solo se per-

3 BAKhtine (1978: 287); itálicas de Bajtín (формами публичного самосознания человека, Бахтин 2012: 394). Cito de la edición francesa porque en varios puntos clave es más fiel que la castellana. Aquí, sin embargo, ambas traducciones optan por "conciencia", pese a la centralidad del concepto de "autoconciencia" (самосознание) utilizado por BAJTín.

4 Ibidem. Con su habitual sutileza, Bajtín no se hace la pregunta típica que, según MoMIGLIANO, sigue siendo la gran incógnita para los estudiosos de la biografía después de Friedrich Leo ("The real question raised by Leo's book is whether we are right in placing on the school of Aristotle the burden of having invented biography", MomigLIano 1993: 20), sino por la indudable presencia de elementos aristotélicos en la biografía. 
fecciona con esta autoconciencia). La vida, concebida de este modo, cuenta con una unidad indudable y se presta para ser representada en una obra de corte clásico, que podrá responder al precepto horaciano simplex dumtaxat et unum.

Sin embargo, la noción idealista de que una vida puede simplificarse en un solo momento o en un solo concepto es problemática desde otros puntos de vista. Cualquier biógrafo podría alegar que devasta la multiplicidad inherente a la persona. Aristóteles mismo constató la irreductibilidad del acaecer sensible concreto a una unidad conceptual y narrativa, motivo por el cual en un pasaje famoso de su Poética niega la universalidad de la poesía a la historia $^{5}$, en particular a la historia de un individuo. En efecto, para ilustrar en qué consiste ese vulgar particular ( $\tau$ ò $\left.\kappa \alpha \theta^{\prime} \varepsilon \tilde{\kappa} \kappa \alpha \sigma \tau o v\right)$ del que se ocupa la historia, Aristóteles pone como ejemplo extremo la biografía: ejemplo de particular, dice, es qué hizo o qué le sucedió a Alcibíades ${ }^{6}$.

La solución de Aristóteles frente a la dificultad de reconducir lo sensible a una unidad dotada de universalidad, entonces, fue descalificar la historia y a fortiori la biografía histórica ${ }^{7}$. Esta crítica, de todos mo-

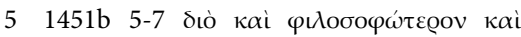

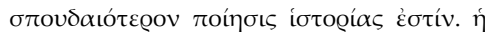

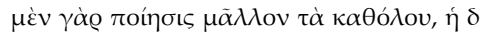

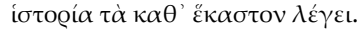

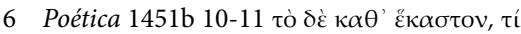

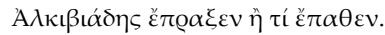

7 Para otros tipos de biografía que el estagirita habría juzgado menos duramente, $\mathrm{cf}$. dos, no puede aplicarse del todo a la biografía antigua y tardo-antigua. Quienes la practicaron en tiempos post-aristotélicos, fueran éstos peripatéticos de segunda o tercera generación, eruditos helenísticos o autores del período imperial, coinciden en que, pese a la multiplici-

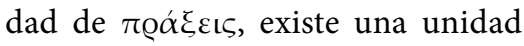
de $\tilde{\eta} \theta$ os. No solo eso: el principio para seleccionar entre la multiplicidad debe buscarse en el carácter; se narrará lo que permita ilustrarlo del mejor modo. Así, Plutarco distingue biografía e historiografía: ou้ $\tau \varepsilon \gamma \dot{\alpha} \varrho$

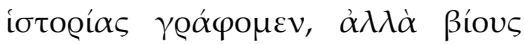
(Alejandro 1, 2); no deben esperarse acciones meramente históricas como tales sino lo que contribuya a pintar el carácter de una persona. Mientras

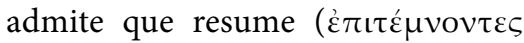
$\tau \dot{\alpha} \pi \lambda \varepsilon \tilde{\tau} \sigma \tau \alpha, 1,1$; cf. Galba 2, 3), no falsifica ${ }^{8}$. Toma parte de una multiplicidad (no el todo), y sin embargo aspira a no tergiversar. El principio en Plutarco es, desde luego, seleccionar lo que mejor pinte un carácter. En términos de LeO (1901: 184-185, en el capítulo sobre la forma biográ-

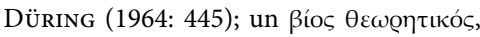

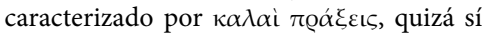
habría sido aceptable, ya que sus exempla tendrían universalidad, mientras su carácter episódico se habría visto mitigado por un tema unificador.

8 También Polibio $(10,21)$ señala que es necesario resumir sin falsear. Manifiesta su preocupación por lo real al señalar que la historia de un individuo se diferencia a la vez del elogio y de la censura y busca la ver-

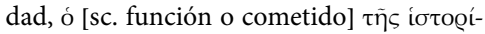

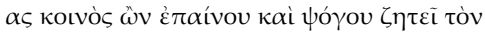

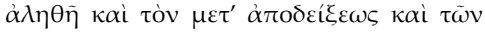

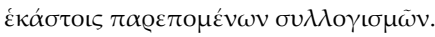




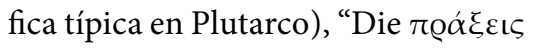
werden geschildert, damit aus ihnen das $\tilde{\eta} \theta$ o $\varsigma$ hervortrete"; en Suetonio, siempre según Leo, se describe el ñOos directamente. Momigliano (1993: 11) observa con acierto: "nobody has ever succeeded in giving, or perhaps even attempted, a complete account of what one man did during his lifetime. But this seems to be the paradoxical character of biography: it must always give partem pro toto; it must always achieve completeness by selectiveness". Esta verdad obvia, conocida por cualquier biógrafo, se convirtió en un tópico en los proemios hagiográficos, donde el autor a menudo observa que se ve obligado a seleccionar solo unas pocas de las múltiples historias que conoce sobre el santo cuya vida escribe; se trata de un locus (ex pluribus pauca, PRATSCH 2005: 31-32). Y el principio de selección, como en Plutarco, suele guiarse por lo que mejor permita pintar un carácter.

\section{La biografía 'clásica' y Friedrich LEO}

$\checkmark$ enofonte narró dos veces la vida de una misma persona, Agesilao: una dentro de una obra de corte cronológico, las Helénicas, y otra en forma encomiástica, elogiando sus diversas virtudes en el Agesilao. Las dos formas no entrañaban una duplicación culpable de contenido, por más que los detalles concretos de su relación sigan generando controversia. Como señala Momigliano, esta doble narración muestra que Jeno- fonte distinguía entre el relato histórico y el elogio'. El primero responde al eje sintagmático, relacionado con la sucesión y la metonimia, el segundo al paradigmático, ligado al tiempo detenido y a la metáfora ${ }^{10}$. En líneas generales, el primero se inscribe en el género histórico y el segundo en el demostrativo o epidíctico.

Se sabe que la oposición más difundida entre dos tipos principales de la biografía antigua se debe a F. Leo (1901). Su punto de vista ha sido criticado ferozmente ${ }^{11}$. Thomas HäGG, por ejemplo, sentencia que su enfoque "was based mainly on formal criteria and inspired by a rigid view of ancient literary genres"; opina que "[it] was largely demolished in the second half of the last century" (HäGG 2012: 232) y que, afortunadamente, "[n]o corresponding theory has replaced it"; esto resultaría conveniente porque su inutilidad "is clear to most scholars". Desde luego, juicios como

9 Momigliano (1993: 50). Jenofonte califica a su Agesilao de Ěracıvos, encomium; MoMIGLIANO observa que su modelo es el Evágoras de Isócrates y que, como Isócrates, "he must have been conscious of turning into prose the traditional poetic eulogy of a dead man". El corolario que MomigliaNo agrega está abierto a discusión: "and he must also have shared Isocrates' belief or illusion that there was no clear link between his encomium and the prose funeral speeches for dead men of earlier times".

10 Cf. JAKoBson (1956: 95-96): "The principle of similarity underlies poetry" (polo metafórico, paradigmático); "Prose, on the contrary, is forwarded essentially by contiguity" y, podríamos agregar, sucesión (polo metonímico, sintagmático).

11 Un útil resumen en González Marín (2000: 83-88). 
los de HäGg pueden recibir la misma crítica que HäGg le realiza a LEo: en particular, que es simplista. HäGG denuesta a LEO por basarse "mainly on formal criteria". La crítica es tan genérica como la de los eruditos soviéticos contra los formalistas rusos. Pero hasta Trotsky le reconoció alguna validez al formalismo: "in spite of the superficiality and reactionary character of the Formalist theory of art, a certain part of the research work done by the Formalists is useful" (cit. en ERLICH 1980: 100.) En verdad HäGG, que mostró su fino olfato filológico en varios libros, particularmente sobre la novela antigua, tiene en mente el carácter esquemático de la teoría de Leo y su falta de plasticidad, no tanto su mentado "formalismo". En efecto, menciona (2012: 68) su "schematizing as such", "the way of looking upon the formation and reproduction of an ancient literary genre as if it had an external history of its own", citando con aprobación a Steidtle: "for the composition of ancient biography the decisive factor at any time is exclusively the special subject, that is, the individual way of life of the person to be described" (HäGg 2012: 68, mis itálicas) Para quien ve el mundo de este modo "cada sol repetido es un cometa". Sobre un positivismo ciego a la importancia de los géneros por el preconcepto de que necesariamente priva de individualidad a las obras particulares, véase Fernández 2020. La influencia del material concreto y de la individualidad no determinan por sí solos una forma, simplemente porque la forma, como el material, no existen de manera independiente ni son aislables. Si a fines analíticos sí puede tal vez separárselos, parece un error de método darle al uno preponderancia sobre la otra sin atención suficiente a su interacción.

Rescatar algunos puntos clave de la teoría de LEo puede parecer anacrónico y sin embargo este es uno de los objetivos de este artículo. Para ello, empiezo por mencionar unas pocas apreciaciones fosilizadas que a menudo se repiten. La contribución de HäGg expone adecuadamente la communis opinio. Así resume este investigador la teoría de Leo (HÄGG 2012: 67-68). Hay dos subgéneros rígidos, el peripatético y el alejandrino. La biografía peripatética se remonta a Aristóteles y sus seguidores; es más artística; se consagra a la vida de figuras político-históricas; está dirigida a una audiencia más amplia; el ejemplo más representativo es Plutarco, con un marco más estrictamente filosófico, una forma más artística y una cronología más estricta. La biografía alejandrina o "gramatical" es creada una generación después por miembros del Museo; es más erudita ("scholarly"), su objetivo es didáctico y se ocupa sobre todo de poetas y filósofos; su ejemplo más representativo es Suetonio, quien usó la forma para sus biografías de gramáticos (a las que se prestaba más naturalmente) y simplemente la trasladó a sus biografías de emperadores. Más allá de un error fáctico (la biografía peripatética se dedicó también a los poetas), es notable que HäGG omite por entero el aspecto que le da interés a la teoría 
de Leo, y que fue, precisamente, retomado por BAjTín en su estudio de las formas del cronotopo: la oposición entre dos modos, ciertamente ideales (y por ende nunca reconducibles por entero a representantes puntuales de un género) de representar la temporalidad.

La idea de que Leo se ceñía rígidamente a uno u otro subgénero es frecuente. WALLACE-HADRILL (1995: 70), por ejemplo, asegura que "Leo's genetic classification never fitted the surviving specimens. Nepos' Lives and Tacitus' Agricola, the main Latin biographies apart from Suetonius, cannot be usefully interpreted in these terms." Sin duda. Pero el mismo Leo (1901: 217-218) lo admitía explícitamente: si bien por su "bello estilo" Nepote se elevaba al modo peripatético, no faltan signos que apuntan "nach der andern Seite" y muestran que la vertiente alejandrina se halla igualmente presente; se trata de un resto "aus der registrirenden Schreibart der grammatischen Biographie, mit deren Mustern sich Nepos natürlich hatte vertraut machen müssen." La rigidez de clasificar en un subgénero en exclusión del otro, por ende, no es practicado por Leo. Quienes se lo achacan deberían explicar pasajes como el recién citado.

Momigliano, más generoso que HägG o Wallace-Hadrill, señala que "[w]hat remains true in Leo's classification is the demonstration that Suetonius was under the influence of an antiquarian approach to biography, whereas Plutarch was nearer to political historiography"
(Momigliano 1993: 19-20). Esta diferenciación genética puede ser crucial, pero conviene poner el acento en aspectos estructurales. Hay dos tipos de biografía, en la perspectiva de Leo (en lo que sigue, modifico libremente su vocabulario): una preponderantemente temporal, otra preponderantemente espacial. Una de sucesión y otra de tiempo detenido. Una más histórica, otra más descriptiva ${ }^{12}$.

En este punto no es ocioso recordar que la oposición entre Plutarco o Suetonio es muy anterior a LeO. Si bien de manera menos sostenida, ya aparece, por dar un ejemplo entre muchos, en Angelo Poliziano, para quien Plutarco o Suetonio no habían aportado menos que Heródoto o Salustio, reconociéndoles a ambos caracteres opositivos capaces de definir rasgos centrales en un sistema ${ }^{13}$.

Ciertamente, la biografía nunca es puramente historiográfica. Los mismos biógrafos antiguos lo saben ${ }^{14}$;

12 En la categorización SonNABEND (2002: 222), "Biographie realisierte sich in der Antike entweder als eine Faktenbiographie oder als eine Charakterstudie". SonNABEND se aleja de Leo en muchos puntos esenciales, pero lo retoma en la recuperación de dos polos opositivos (aunque el contenido de estos polos no sea idéntico en ambos autores).

13 Véase Godman (2019: 55), con las citas originales y bibliografía.

14 Wallace-Hadrill (1995: 10). Cf. la constatación de Leo sobre la mezcla de rasgos genéricos: "Sallust hat biographische Elemente in die Historie, Tacitus historische Elemente in die Biographie hineingearbeitet. Sallusts bellum Iugurthinum und de Catilinae coniuratione sind keineswegs Biographien, wie es der Agricola ist. Nicht Iugurtha steht im Mittelpunkt, sondern Rom 
cualquier carácter, que tras la muerte de un sujeto puede interpretarse como relativamente unitario, tiene elementos extratemporales. Incluso la biografía peripatética intenta dar cuenta de un $\tilde{\eta} \theta$ os que puede describirse relativamente separado de su desarrollo, si bien se manifiesta paulatinamente en el tiempo ${ }^{15}$, motivo por el cual -y esto es crucial- la cronología nunca es minimizada ${ }^{16}$. Esto no significa, como observa MomiGLIANO, que la sucesión temporal sea el único principio ordenador, como en los anales: "Weizsäcker observed that even the Plutarchean type was not entirely organized in chronological order-which is true- and concluded that therefore it is not essentially different from the Suetonian type -which does not follow" (MomigLiANo 1993: 19 ${ }^{17}$. En cualquier caso, la biografía peripatética no deja de lado

und die Nobilität" (Leo 1901: 232). Lo mismo puede decirse sobre el Evaristo Carriego de Borges: no versa sobre el individuo del título sino sobre un barrio porteño y sus personajes típicos, especialmente los marginales; cf. supra, n. 1.

15 "[...] der Charakter wurde nicht in einer eignen Schilderung gezeichnet, sondern er trat allmählich, wie es die peripatetische Ethik lehrte, in den Handlungen des Mannes hervor" (Leo 1901: 317).

16 Aunque pudiera utilizar libremente anécdotas encontradas aquí y allá (como los alejandrinos), la biografía peripatética lo hacía "ohne doch das von Aristoteles angelegte chronologische Gebäude auszubauen" (Leo 1901: 318).

17 Más crudo es Stadter (1989: lii): en Plutarco, "[c]onsiderations of chronology are secondary to those of character and subject", que en esto coincide con las observaciones del mismo autor. la relación entre la historia externa y la vida de un individuo ni el entramado cronológico dentro de la vita. Su temporalidad, que no es la histórica ni mucho menos la analística, tampoco se opone a estas. Momigliano vio con claridad la importancia de la cronología y de la sucesión en este tipo de biografía, tal vez, incluso, sobreestimándola ${ }^{18}$.

La temporalidad de la biografía alejandrina, en cambio, no solo es distinta de la analística sino que constituye su contrapunto estricto: "Die Lage der Dinge zeigt deutlich dass Sueton, wie bemerkt, die Annalistik durch sein Buch nur ergänzen wollte" (Leo 1901: 32). En términos de WALLACE-HADRILL, quien extrañamente alega estar refutando a LEO, "Suetonius establishes the independence of his genre by distancing himself from history the further, the more his subject matter brought him up against historical material. His Caesars were to be in technique a mirror-image of history" (WALLACE-HADRILL 1995: 10). Esto es, esencialmente, lo mismo que propone Leo. Wallace-HADRILL asigna a Suetonio la predominancia del elemento paradigmático, sin sucesión, señalado más arriba como uno de los polos a los que tiende la biografía: "Rather than let biography become history, he [Suetonius] would write non-history" (WALLACEHADRILL 1995: 9); más sutil es lo que propone después, a saber, que escribe el contrapunto (a mirror-image)

18 "The Plutarchean type is a straightforward chronological account of events", МомIGLIANO (1993: 18-19). 
de la historia, no una anti-historia. Así, incluso los críticos de Leo suelen basarse en su enfoque, aunque lo modifiquen en la formulación o lo flexibilicen; rara vez en la esencia que, desde una lectura posible, consiste en subrayar la doble vertiente en la que se inscribe la biografía antigua: historiográfica vs. retórica desde un punto de vista; de investigación histórica vs. anticuarianismo desde otra (véase la siguiente sección con la aportación de Momigliano sobre el tema); con tiempo móvil o tiempo detenido.

\section{La biografía y el cronotopo de Mijaíl BAJTín}

B ajtín escribió su contribución sobre los cronotopos $^{19}$, inédita por cuatro décadas, en los años 30. Como es típico en él, jamás menciona a Leo; lleva a cabo la misma omisión de fuentes secundarias que explicita en "El problema del contenido": las citas son inútiles ya que el lector erudito no las necesita, y para aquel que no lo es son vanas (BAKHTINe 1978: 23).

Esta omisión causó un serio perjuicio a la comprensión genética de su monografía. Casi no se reconoce la semejanza estructural entre sus cateogorías de biografía antigua y las de LEo. Cuando sí se lo hace, suele ser de

19 Definidos como "la interconexión esencial de las relaciones espacio-temporales tal como han sido asimilada por la literatura" (ВАкнтіNe 1978: 237); Существенную взаимосвязь временных и пространственных отношений, художественно освоенных в литературе (Бахтин 2012: 341). modo incompleto o errado. Un ejemplo es VAN WaARden, quien menciona muy al pasar a Leo tras aplicar el cronotopo bajtiniano a Sidonio Apolinar. Tan al pasar lo hace, efectivamente, que asocia a Suetonio con el método peripatético ${ }^{20}$. Esta confusión está en parte motivada por el secretismo de Bajtín sobre sus fuentes. HirschKop (2001: 3) lo señala con claridad: "the unacknowledged source for much of Bakhtin's commentary on ancient literature is Georg Misch's Geschichte der Autobiographie." Agrega en otra publicación: "The section of 'Forms of Time and of the Chronotope' on ancient autobiography [en realidad, sobre todo el capítulo, incluyendo la biografía] follows MiscH in its choice of authors, in its description of practices, and, most strikingly, in its classification of types of autobiography"; sigue una referencia a Brian Poole, quien descubrió que en el texto mecanografiado de "El discurso novelesco" BAjTín llama la atención sobre el capítulo de Misch acerca de la "auto-representación en las formas realistas de la literatura"

20 VAN WAarden (2020: 164, n. 79): “This distinction [entre biografía 'energética' y 'analítica'] goes back to Friedrich Leo's seminal book Die griechisch-romische Biographie nach ihrer litterarischen Form (Leo [1901] 1965), in which he distinguishes Suetonius's record-keeping style, set out according to subject matter and influenced by the peripatetic empirical method of collecting material, from Plutarch's essentially chronological take, where a character becomes manifest in deeds, following Aristotle's concept of èthos (ท̃ $\theta 0 \varsigma)$ "; posiblemente en lugar de "peripatetic" deberíamos leer "antiquarian". 
(HirschKop 1999: 232, n. 10). La edición rusa de las obras completas demuestra lo que HirschKop y Poole entreveían: incluye un ensayo sobre el libro de Misch con largas citas en alemán acompañadas por numerosas notas de BAjтín (Бахтин 2012: 669708). El capítulo sobre el cronotopo biográfico está imbuido de categorías de Leo, independientemente de que estas hayan sido tomadas de $\mathrm{MISCH}^{21}$, quien desde luego conocía bien a LeO y buscaba, entre otras cosas, colmar las ciertas lagunas de este último en el ámbito de la autobiografía antigua ${ }^{22}$. La definición de Misch de la autobiografía como una "historia de la autoconciencia humana", Geschichte des menschlichen Selbstbewusstseins, puede tener sus raíces inmediatas en Dilthey, como observa MomigliaNo (1993: 18); para Dilthey la autobiografía, en tanto Selbstbesinnung de la persona sobre su vida, estaba en la base de cualquier concepción histórica, cfr. Dilthey 1965: 201. Pero sus antecedentes explícitos pueden rastrearse también en Schelling, en cuyo System des transzendentalen Idealismus (1800) la filosofía es concebida, precisamente, como una "historia de la autoconciencia” (DRÜE 1994: 292). Esta idea también es crucial en las

21 Misch 1907. Cito de esta primera edición por ser la que conocía BAjTín y porque sus rasgos distintivos aparecen allí con mayor claridad que en la reedición aumentada de 1949-1950.

22 Sobre la idea de Leo de que los griegos no conocían la autobiografía, aparentemente debida a su negativa a tomar en cuenta el material que se halla fuera de la biografía formal, cf. Momigliano (1993: 15).
"Formas del tiempo y del cronotopo en la novela", cuyos elementos kantianos (y tal vez hegelianos) todavía no han merecido un estudio exhaustivo; recuérdese la importancia de la "autoconciencia del hombre público" (supra, n. 3).

Según Bajtín, que en esto, como hemos visto, no es original (supra, "La biografía 'clásica”'), existen dos grandes modelos biográficos antiguos, a saber Plutarco y Suetonio. En el primero, "el tiempo biográfico es específico: es el tiempo de la revelación del carácter, pero de ningún modo el del devenir y el crecimiento. El tiempo es fenoménico, la esencia del carácter es intemporal [...] la realidad histórica misma [...] no sirve más que de medio concreto a esta revelación" ${ }^{\prime 23}$. Un carácter está dado desde el principio, y el objetivo del biógrafo es describirlo según sus rasgos intemporales que, por lo demás, servirán de modelo a seguir (o rechazar) para generaciones posteriores $^{24}$. Las vidas de Plutarco, de este modo, tienen una esencia, oủoía, y pueden definirse -de modo aristotélico- según género y dife-

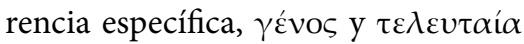

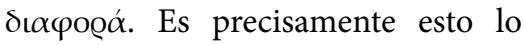
que autoriza la célebre comparación

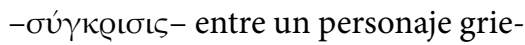
go y otro romano. Vidas puramente históricas no lo habrían permitido. Con esta forma se relaciona la cita de Borges según la cual una vida entera se define por el momento en que el

23 BAKHтine (1978: 288; Бахтин 2012: 395).

24 Cf. supra, "La unidad, lo universal, lo sensible", sobre el método de Plutarco expuesto en Alejandro. 
hombre sabe para siempre quién es. En Plutarco, de todas maneras, el carácter único se revela paulatinamente en el devenir histórico particular y, en esta misma medida, la sucesión sí tiene una importancia determinante $^{25}$. Recordemos la observación de Momigliano: "The Plutarchean type is a straightforward chronological account of events" 26 . En este punto, la biografía se encuentra anclada en el eje de lo sintagmático, aunque haga foco en el punto central de una vida, el carácter, que sería único y existiría fuera del tiempo ${ }^{27}$.

Suetonio, por el contrario, se apoya poco en la sucesión, salvo al comienzo y al final de las vitae; este marco se repite en muchas obras, no solo biográficas, que comienzan por el nacimiento o la infancia y terminan con la muerte y en cuyo interior se suceden anécdotas desconectadas de cualquier cronología. Estudia las vidas según categorías atemporales fijas, como la vida social, la vida familiar, las virtudes, los vicios, etcétera. Este tipo de biografía analítica se sitúa de preferencia en el eje de lo paradigmático.

25 Cf. supra, n. 16.

26 Momigliano (1993: 18-19). Hemos visto que diversos autores le dan distinta importancia a la temporalidad, la sucesión y la cronología en Plutarco, pero todos coinciden en otorgarle alguna (mayor, en contraste, que la presente en Suetonio).

27 Sobre la relación entre un ethos relativamente separado del tiempo, aunque se revele en él, y las praxeis plenamente temporales, cf. supra, "La unidad, lo universal, lo sensible". Sobre la importancia de la cronología, cf. supra, "La biografía 'clásica".
En cualquier caso, ciertas continuidades opositivas se mantienen: originalmente había dos vertientes en dos géneros distintos, el historiográfico (sintagmático, con sucesión) y el retórico-demostrativo (paradigmático, de tiempo detenido). Jenofonte narra la vida de Agesilao según la primera vertiente en las Helénicas, según la segunda en el Agesilao. WallaceHADRILL (1995: 144), tras fustigar a LEO asegura esto mismo: "Suetonius' approach, therefore, is not (as Leo believed) the product of any biographical tradition". Aquí podríamos decir, con Momigliano: es directamente el producto de un enfoque, el anticuario (véase el siguiente párrafo), e indirectamente de un tipo de biografía influido por este enfoque, el de las vitae de poetas. WALlACE-HADRILL continúa: "The literary parentage of this aspect of the Caesars is a tradition going back to Xenophon's encomium of the Spartan king Agesilaus." Con esto, Wallace-Hadrill redescubre lo que un lector atento de LEO o de Momigliano ya sabía: la biografía que tiende al polo de lo paradigmático tiene sus raíces en la retórica epidíctica.

Las dos vertientes o polos, que tienen su tipo ideal (imperfectamente histórico) en las biografías de Plutarco y Suetonio pueden clasificarse también de otra manera: no por género sino por método. Esta tentativa se debe a Arnaldo Momigliano. Como Leo, Momigliano hace una división binaria y opone (sin llamarlos de ese modo) los polos sintagmático y paradigmático: 
[...] the Greeks distinguished between history and erudition, between what they called historia and what they called less clearly and less unequivocally archaeologia or philologia and the Romans translated as antiquitates. The distinction was by no means sharp and self-evident, but it did exist and I was able to trace its development in an old lecture of mine at the Warburg Institute ${ }^{28}$. The basic distinction between the two subjects was that history dealt mainly with political and military events and was written in a chronological order, whereas erudition dealt with almost anything else -from personal names to religious ceremonies- and preferred systematic survey to chronological order.

La biografía sintagmática está influida por la historia propiamente dicha, la paradigmática por el anticuarianismo $^{29}$. Evidentemente, si bien

\section{Momigliano 1950.}

29 Las clasificaciones binarias de la biografía abundan. No todas son igualmente útiles. Recientemente, sobre líneas de posicionamiento frente a los valores morales y sociales predominantes, Konstan \& WALSH (2016) diferencian la biografía cívica o defensora de valores oficiales de la "subversiva" o contracultural, que subraya el valor del ingenio (wit) y demás recursos de los desposeídos (2016: 43); más polémicamente, consideran que la primera se presta más al relato cronológico que la segunda (2016: 43). Encuentran ejemplos de ambas en Jenofonte: Agesilao para la primera, Sócrates en los Memorabilia para la segunda. Los cínicos apreciaron esta visión subversiva de Sócrates (2016: 34). Otros ejemplos: la Vida de Esopo, el Romance de Alejandro, los evangelios. Con una argumentación poco feliz, la Vita Antonii de Atanasio de Alejandría se considera representativa de una "síntesis cristiana" entre las dos tendencias, la representación de Agesilao en las Helénicas está de pleno en el ámbito de la historia, el Agesilao, asociado a la retórica, tiene poco que ver con el anticuarianismo. Sin embargo, sí está asociado al anticuarianismo en un punto clave, precisamente la relativa irrelevancia del paso del tiempo y de la cronología.

Sabemos que Leo (seguido por Misch, BAjtín y muchos otros) divide a la biografía, afianzada como género separado, en dos tipos principales: el peripatético y el alejandrino. Podemos agregar que cada uno de ellos, ejemplificados por Plutarco y Suetonio respectivamente, sigue de preferencia el eje sintagmático o el paradigmático. Existen, sin duda,

\footnotetext{
"a narrative form that emphasizes a new set of virtues. As such, the lives of Christian heroes came to resemble the old civic model, but in a new key" (2016: 43). Esta conclusión parece incompatible con las líneas principales de su artículo; en un mundo ideológico cambiado, la figura del héroe es distinta sin que esto implique subversión. Más adecuado a sus fines hubieran sido la Vida de Simeón el Loco de Leoncio de Neápolis, las anécdotas de los Apophtegmata Patrum o, en el Occidente latino, la pasión de Maura, esposa de Timoteo (Bibliotheca Hagiographica Graeca 1848z, 1849), que se burla de que el agua en la que la queman está fría, haciendo caer al propio gobernador en la trampa de tocarla (el relato más difundido es el mencionado por Curtius (1948: 427), con ejemplos semejantes de Ambrosio de Milán y de Prudencio; en la opinión de Curtius, sin duda errada, se deben a la tradición romana). Sin llamarlo de ese modo, Konstan \& Walsh ponen el acento en el desbordamiento de límites genéricos tradicionales proporcionados por la figura de un trickster.
} 
vitae mixtae ${ }^{30}$. Aunque algunos las toman como una refutación del sistema de Leo, la división de este autor no es absoluta; se trata de un instrumento de análisis, no de un lecho de Procusto para encuadrar de manera tajante todas las biografías conservadas ${ }^{31}$. Los polos ideales son de utilidad para señalar rasgos en una vita dada, no para ubicarla en un compartimento estanco.

30 Para una breve caracterización crítica de LEO, especialmente por remisión a las vitae mixtae, cf. VAN UYTFANGHE (2005: 227229). En una vena semejante, autores como HäGG (2005:68) consideran que el hallazgo papiráceo de la biografía de Eurípides en verso, debida a Sátiro (publicada en 1912), difícilmente clasificable como peripatética o alejandrina, muestra que la teoría de Leo es errónea. El hallazgo es posterior a la publicación del estudio de LEO; difícilmente el autor hubiera podido tenerla en cuenta, si bien es cierto que llevó a cabo una defensa del carácter "alejandrino" de Sátiro (anterior al hallazgo del fragmento papiráceo) que no convence; véase WEST (1974: 280): Ateneo llama a Sátiro "peripatético" pero LEO estima que esto no debe entenderse de modo literal, tratándose únicamente, en su visión, del momento en que la biografía peripatética deviene alejandrina. "Von den drei männern, die in Alexandria selbst den Uebergang von der peripatetischen zur alexandrinischen Biographie vermitteln, heissen zwei Peripatetiker, dem dritten hat man den Namen geben wollen. Vom Peripatos zeigen sie nichts, als den Typus der litterarhistorischen Studien" (LEO 1901: 118). Esta excentricidad de negar a las palabras su sentido habitual sin justificar por qué reciben otro nuevo no implica, desde luego, que las bases de su sistema estén comprometidas.

31 Cf. supra, "La biografía 'clásica", para una discusión de Nepote considerado según rasgos peripatéticos y alejandrinos por el mismo Leo.
Debe recordarse que algunos autores ponen en entredicho que la biografía sea un género mixto; una obra que se relacione con la retórica epidíctica no podría pertenecer al género biográfico. En su introducción a la Vida de Macrina de Gregorio de Nisa, por ejemplo, Maraval menciona a quienes le negaron a esa obra el carácter de "biografía" o de hagiografía, al considerarla una oración fúnebre. Según Maraval, que en este punto sigue a G. BARDY, la biografía y la hagiografía se caracterizan por estar destinadas a ser leídas, mientras la oración fúnebre o el elogio deben ser pronunciadas ${ }^{32}$; por ese motivo, la Vida de Macrina sería hagiografía.

A. Dihle indica que muchos estudiosos, perdiendo la esperanza de una definición precisa frente a la multiplicidad de formas involucradas, negaron la posibilidad de definir la biografía con relación a un género y remitieron a la teoría de géneros antiguos, en la que la biografía no juega ningún rol $^{33}$. Quizá sea conveniente, con VAN Uytfanghe, admitir que "lécriture biographique (la Personendarstellung comme on dit en allemand) est une écriture très ouverte et flexible, qui entre facilement en osmose et en recoupement: l'historiographie, l'encomion [...], le roman ou la nouvelle [...], sans parler de l'arétalogie, encore plus

32 Maraval 1971: 22-23. Sobre el tema de los géneros, cf. Ferrer (2013: 319-320). BeCK (1959: 268), por su parte, asegura que los panegíricos y encomios auténticos no corresponden propiamente a la homilética, sino a la hagiografía.

33 Dihle (1998: 121). 
chimérique" ${ }^{\text {34 }}$. Esta fluidez dificulta la clasificación genérica.

La discusión acerca de si los diversos representantes del discurso hagiográfico (infra, "La hagiografía") constituyen stricto sensu un género no es central para esta contribución. Sí lo es, en cambio, la función de los polos sintagmático y paradigmático.

\section{La hagiografía}

C xisten obras típicas de lo que, para evitar pronunciarse sobre la existencia de un género, VAN UytFAnghe (2011: 36-37) llama "discurso hagiográfico" (esta denominación aparece ya en de CerTeAu (1968: 207); incluye obras paganas, cuya mención omitimos:

[...] on peut citer la Vie de Constantin et celle d'Origène par Eusèbe de Césarée (†339), la Vie d'Antoine par Athanase d'Alexandrie ( $\uparrow 373)$, les Vies de sa soeur Macrine et de Grégoire le Thaumaturge par Grégoire de Nysse $(† 395)$, loraison funèbre pour saint Basile par Grégoire de Nazianze ( $\dagger$ 390), la Vita prima grecque de Saint Pachôme (vers 400), la Vie de Martin de Tours par Sulpice Sévère ( $\dagger$ vers $420)$, les Vies de moines par Saint Jérôme $(\dagger 419 / 420)$ et bien d'autres. [...] on confectionne également des "biographies collectives": [...] l'anonyme Historia monachorum in Aegypto (vers 400) et l'Histoire Lausiaque par Palladius d'Hellénopolis ( $\dagger$ peu av. 431) [...] (sin itálicas, salvo en el caso de la Vita prima y la $H M A$, en el original).

34 VAN Uytfanghe (2005: 227).
Las definiciones de la hagiografía (y de la hagiología, que en ocasiones se le diferencia) abundan. También las obras que incluye: son generalmente aceptadas vidas de santo, actas de mártires, translationes reliquiarum, colecciones de milagros, mientras que compilaciones de dichos como los apophtegmata patrum tienen una posición más ambigua. En cualquier caso, la unidad genérica de la hagiografía es discutida: desde las passiones auténticas hasta las vitae en estilo elevado hay numerosos matices; ni siquiera su carácter literario está asegurado, mucho menos una estructura composicional estable, un mismo patrón de espacialidad o temporalidad, un rol semejante del narrador, etcétera. Es frecuente en la literatura especializada argumentar que la hagiografía solo puede definirse semánticamente, por su contenido. Si eso fuera verdad, desde luego no se trataría de un género. Estas complejidades no afectan las líneas generales de este artículo y no serán discutidas $^{35}$, salvo en un punto nodal: precisamente, la presencia de los polos sintagmático y paradigmático. Al menos para contar con un marco conceptual bien establecido, la contribución de Leo sigue resultando instrumental, del mismo modo que el debate sobre si la hagiografía era predominantemente "suetoniana", aunque haya caído en descrédito (GONZÁlez MARÍn 2000: 87), aclaró muchos procedimientos compositivos de los hagiógrafos.

35 Véase GonZÁLez Marín (2000). 
Numerosas vidas de santos combinan sucesión y tiempo detenido en el interior de una misma obra. Cuentan con una sección que presenta hechos puntuales, acaecidos en un lugar preciso y ordenados según el antes y el después, contrapuesta a otra sección de temporalidad débil sobre las características del santo (su misericordia, su clarividencia, etcétera), a menudo ejemplificado con aventuras que pueden tener el carácter de exem$p l a^{36}$. Hay innumerables variaciones, pero la oposición suele verificarse. Un ejemplo entre muchos aparece en la Vita Spyridonis, en versión de Simeón Metafrastés: la vida de Espiridón, dice el autor, está suficientemente testimoniada por los milagros que ha relatado; ahora se hace necesario incursionar en su celo en torno a la fe ${ }^{37}$. Aquí, Bíos

36 En ocasiones, la ejemplaridad puede arrasar con cualquier registro histórico. En la recensión del Parisinus lat. 3820 de la Vida de Juan el Limosnero de Leoncio de Neápolis, el carácter de Juan, patriarca de Alejandría, se angosta hasta casi desaparecer, transformándose en una ficelle que une diversos episodios en los que aparece su generosidad y su afición por la limosna. De individuo pasa a tipo (el Limosnero); solo se incluyen las historias que ponen de relieve ese rasgo. Aquí el carácter (resumido en un único rasgo) se manifiesta una y otra vez en aventuras que, en cierta medida, no

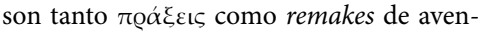
turas precedentes. Aquí la temporalidad se acerca aún más a la de las fábulas esópicas o los cuentos populares y esto, al menos en parte, es determinado por el tipo de protagonista (que tiene una influencia directa en la estructura composicional).

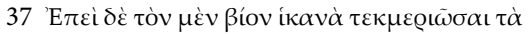

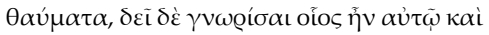

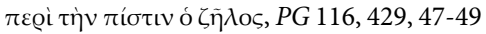
(Migne). y $\theta \alpha u ́ \mu \alpha \tau \alpha$ se oponen a $\zeta \tilde{\eta} \lambda$

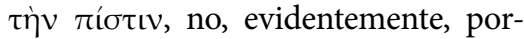
que sean incompatibles, sino porque son puntos de vista distintos sobre una unidad que la hagiografía busca resaltar. En otras vitae hay esquemas bipartitos análogos. Los títulos -Bíos

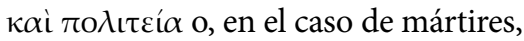

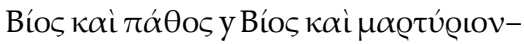
responden a la bipartición, si bien no de modo idéntico: ßíos representa, en líneas generales, el relato cronológico, $\pi 0 \lambda \iota \tau \varepsilon i ́ \alpha$ el sistemático, y en su conjunto pretenden ofrecer un panorama completo de la vida del santo o, diríamos nosotros, de su "vida y obra". El esquema ha tenido una gran continuidad léxica, aunque su significado haya mutado: en la Grecia contemporánea, The Life and Times of Scrooge McDuck (Rico McPato) se traduce como $O$

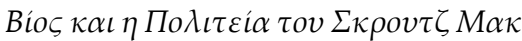
$N \tau \alpha \kappa$. Menos codificado, ya existía en la biografía antigua, como señala LeO al pasar, la división entre "ßíos und $\alpha \dot{\pi} \sigma \varphi \theta \varepsilon ́ \gamma \mu \alpha \tau \alpha$ " (1901: 183) o entre

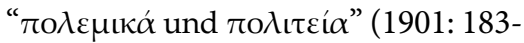
184).

En cualquier caso, el relato separado y episódico de milagros que podrían sucederse casi en cualquier orden constituye en gran medida un subgénero mixto, ya que si bien cada episodio está motivado témporo-causalmente en su interior, su relación con el mundo cronológico del relato biográfico total suele ser oscura, y en ciertas vidas solo el comienzo y el final siguen de manera clara una estructura temporal de primeros años-desarrollo-muerte. Esta estructura episódica no es exclusiva de la 
hagiografía, desde luego; se verifica en muchas biografías "anecdóticas", como las de Diógenes Laercio, la Vita Aesopi, etc., y su estructura externa tiene que ver también con la biografía de Suetonio ${ }^{38}$.

\section{El cronotopo de la hagiografía y su relación con el biográfico}

(J no de los empleos más astutos del cronotopo biográfico de Bajtín fue llevado a cabo por Stadter quien, en su análisis de la Ciropedia (1991: 474), escribe que esta obra presenta cuatro fases, una dinámica (la juventud y el crecimiento, libro I) y tres "not developmental but static", sin crecimiento ni desarrollo (campañas militares y ascenso al poder; retribución por la virtud del monarca; testamento y muerte, libros II-VIII) (StAdTER 1991: 473-477). La división ha sido criticada por HäGG (2012: 54): "It appears strange [...] to read the bulk of the work as 'static' or 'timeless'. Even though chronological markers are largely missing, there is throughout a sense of chronological succession, not least through the constant growth of Cyrus' empire." HäGG parece no tener presente el objetivo de STADTER: describir la ausencia de efecto sobre el personaje principal del paso del tiempo, no la detención categórica del tiempo. "Cyrus has not in fact changed in the intervening books [entre finales del libro I y

38 Tal vez este es el motivo por el que varios autores encontraron una influencia específicamente suetoniana en la hagiografía; cf. GonZÁlez MARÍn (2000: 87). el VIII]" (Stadter 1991: 474); Ciro no cambió, pero los años ciertamente transcurrieron (esto no significa que todos se narren; STADTER sugiere que hay una elipsis entre los treinta o treinta y un años de Ciro y su muerte a una edad mucho más avanzada; 1991: 477, n. 39). Es poco feliz que STADTER no subraye el carácter mixto de esto temporalidad, que no es simplemente un "tiempo biográfico", como la llama STAdTer. En muchos puntos se acerca a la que BAJTín asigna al "tiempo de aventuras" de la novela griega, uno de los aspectos más polémicos de su teoría del cronotopo, que transcurre entre que los héroes se conocen y consuman su matrimonio. Sería una pausa, un hiato fuera de la serie biográfica temporal (BAKHTINE 1978: 241-242) en donde los héroes no cambian ni se transforman, ni siquiera envejecen (1978: 242). BAJTíN podría asegurar, como STADTER sobre Ciro, que los protagonistas "have not in fact changed in the intervening books". Ciertamente, tampoco suelen tener un carácter reconocible y diferenciable como el de los protagonistas de las biografías de Plutarco o Suetonio, y en este punto el Ciro de Jenofonte sí se acerca al cronotopo de la biografía y la autobiografía antiguas. La presencia de este cronotopo híbrido quizá habría contribuido a la polémica postulación de STADTER de que la Ciropedia es "ficción" y, más específicamente, una ficción biográfica: combina, en efecto, elementos reconocibles de dos temporalidades, una novelesca, la otra biográfica. STADTER, por lo demás, cita a BAJTín solo 
en referencia a la biografía peripatética, no a la alejandrina, con la que el esquema de la Ciropedia tiene mucho en común.

BAjTín exagera el carácter relativamente estático y abstracto del tiempo de la novela griega y de la biografía antigua en parte por una estrategia discursiva: subrayar, por contraste, los cambios que sí son visibles en la narrativa posterior (como el Bildungsroman, el Entwicklungsroman y sus variantes paródicas como el Candide de Voltaire), poniendo el acento en un aspecto diferencial del tiempo de aventuras. En cualquier caso, y con la excepción de Dafnis y Cloe, en la novela griega hay una temporalidad paradójica que tiene puntos de contacto con la de la biografía analítica ${ }^{39}$ y con la de la hagiográfica. Dentro de cada episodio específico (por ejemplo, el ataque de los piratas o la tempestad en el mar en el caso de la novela griega) el tiempo ciertamente transcurre, por más que haya atrofiamiento y casi ausencia de sucesión en el tiempo global ${ }^{40}$, con perso-

39 Incluso si se postula que en esta biografía no predomina la sucesión, habría que calificar la afirmación: en la estructura global, tal vez (con la escasa importancia de la cronología y de la historia externa, salvo en el comienzo y el final de una vita), pero no en el interior de los episodios, muchos de los cuales son anécdotas con principio, nudo y desenlace plenamente temporales. Véase la próxima nota.

40 Si el tiempo transcurrido no deja huella en los protagonistas (contra LALANNe 2006), la duración sí cuenta "dentro de los límites de cada aventura", donde "hace falta tener el tiempo para huir, para alcanzarse, para distanciarse"; allí la cronología es central: najes que se ven poco afectados por el tiempo o por el entorno; quizá esto es lo central: no solo la ausencia de un cambio motivado por el tiempo, con madurez y crecimiento, sino también la relativa impermeabilidad a las circunstancias, que en una literatura posterior contribuyen a moldear de manera específica los caracteres: una aspecto crono-tópico, no únicamente temporal. Algo semejante sucede en la Ciropedia. Un cronotopo semejante al suyo, en el que se liga el tiempo abstracto con un cambio reconducible a una prueba concreta, a un solo momento de cambio profundo ${ }^{41}$, o a

hasta los minutos o los segundos pueden ser cruciales (BAKнtine 1978: 244). Hemos visto que lo mismo sucede con muchos episodios de la biografía analítica o de la hagiografía. Ninguna narración combina un tiempo global moroso (o detenido) tanto en el todo como en las partes, al menos hasta la novela bizantina (s. XII), donde en los episodios mismos suele predominar un tiempo perezoso: por ejemplo, una larga écfrasis o el recitado de una larga canción. La novela bizantina es una de las primeras manifestaciones de esta rareza narrativa donde el tiempo coagula tanto en el todo como en las partes.

41 En la Ciropedia, "the long dialogue with his father which ends the first book establishes Cyrus' maturity and his understanding of the characteristics necessary in a leader" (STADTER 1991: 774). Esa charla perfecciona el rito de pasaje; Ciro cambia de una vez y para siempre. El evento único que es el punto de giro de una vida aparece también, sin que haya desde luego ninguna influencia directa, en muchas vidas de santo. Por dar un ejemplo: Atanasia de Egina descubre su vocación monástica cuando una estrella brillante desciende hasta su seno (ó@õ $\varphi \varepsilon \gamma$ -

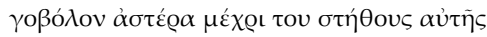
$\kappa \alpha \theta t \varepsilon ́ v \tau \alpha)$ (CARras 2017: 212, § 3); San Martín, en la hagiografía de Sulpicio, en la 
un período circunscripto de cambio, sin evolución progresiva ni constante, aparece en varias vidas de santo. Tal como sucede con gran parte de las novelas griegas, la temporalidad de la Ciropedia y la de cierta hagiografía es la del Prüfungsroman, la novela de prueba; hay un rito de pasaje (cf. LALANNE 2006), pero habitualmente no una progresiva evolución en el tiempo de un carácter. ${ }^{42}$

Un ejemplo es la Vida de Simeón el Loco de Leoncio de Neápolis (CAVALLERO 2009): el protagonista conoce a su amigo Juan durante la exal-

noche posterior a partir su manto con un mendigo (cap. 3, ed. FonTAINE) tiene una visión en la que Jesús, rodeado por un coro de ángeles, le dice que él, Martín, aunque es apenas un catecúmeno, lo ha vestido; Martín, por entonces de veinte años, se apresura a recibir el bautismo. Cambios radicales que motivan conductas posteriores abundan también en el Nuevo Testamento: piénsese en Pedro que, arrepentido por negar tres veces a Jesús y fortalecido por esa debilidad que no volverá a conocer, encuentra la fuerza para soportar el martirio. Otro ejemplo es el modelo narrativo de las Confesiones de Agustín (ed. Verheijen 1981), que giran en torno a la conversión al cristianismo; sólo en esa medida interesa todo lo que la prefigura, como la conversión a la vida filosófica con la lectura del Hortensius de Cicerón. Se trata, en términos borgeanos, del momento en que alguien sabe para siempre quién es.

42 Pese a las críticas su postulación de contra una temporalidad estática en el tiempo de aventuras, BAjTín tiene presente en $\mathrm{El}$ discurso novelesco que el Prüfungsroman (en el que subsume, mutatis mutandis, a la novela griega) puede implicar una crisis y regeneración (BAKHTINE 1978: 206) o, diríamos nosotros, un rito de pasaje. Lo mismo sucede con gran parte de la hagiografía; cf. infra. tación de la cruz en Jerusalén, "en tiempos del emperador Justiniano"; ambos admiran a los monjes que habitan a lo largo del Jordán ("ángeles del cielo" según Juan) y deciden emularlos. Existe una prolepsis: Simeón saca "diez más que Juan" en un misterioso juego, prueba de que lo superará en ascesis y virtud. Pasan tiempo en monasterios, se tonsuran, viven por veintinueve años en el desierto, donde superan todo tipo de mortificaciones y pruebas y alcanzan un alto grado de perfección -sobre todo Simeón, que decide volver a la ciudad, en este caso Emesa. Hasta aquí hay un tiempo biográfico específico, que saca a la luz algo precognoscible (la virtud superior de Simeón) pero no inmutable ni preexistente como los caracteres de la biografía antigua según los entiende BAjTín, modificado también por un entorno específico (Jerusalén, los monjes del Jordán). De ahora en más, un Simeón que ya alcanzó la perfección lleva a cabo milagros. En algún momento se confunde o piensa no estar a la altura de sus fines; sin embargo, en las aventuras de Emesa el tiempo transcurre solo en el interior de los episodios, que podrían sucederse casi en cualquier orden; en ellos Simeón no parece envejecer ni cambiar, como sí cambió en la sección anterior. El desarrollo llegó a su fin. Los episodios muestran, en un tiempo general detenido, la virtud específica del santo, sus principales características: ser absolutamente impasible, por un lado, y simular locura para que no se reconozca su virtud, por el otro. Finalmente le llega la 
muerte: no porque haya transcurrido un determinado número de años sino, podría decirse, porque no quedan más anécdotas que relatar.

En otra vita de Leoncio de Neápolis (CAVAllero 2011) el procedimiento es más simple: los años de formación y cambio de Juan el Limosnero son tácitamente pasados por alto; los episodios se ordenan desde el inicio paradigmáticamente, por tema, en torno a la virtud del patriarca, su generosidad, su pasión por las limosnas, etc. El reverso de esta temporalidad es la de la Vita Antonii de Atanasio de Alejandría (BARTELINK 1994), cuyo protagonista es afectado por el paso del tiempo y sigue siendo sometido a pruebas, al menos mínimamente, incluso luego de períodos de cambio como los veinte años de soledad y ascesis en el desierto. En efecto, Antonio retorna a la ciudad, pero cuando teme que se lo estime demasiado parte hacia la "montaña interior", donde vuelve a vivir en soledad, como en sus años en el desierto, y alcanza un mayor grado de perfección. A diferencia de Simeón o de Juan, su proceso en pos de las virtudes es más sinuoso y extenso; su cronotopo es más estrictamente biográfico.

\section{Conclusiones}

(1 l igual que la Ciropedia, la hagiografía combina dos cronotopos diversos, el de la novela griega y el de la biografía antigua. Este cronotopo mixto se condice con la difícil caracterización de un género (o de un discurso) como el hagiográfico, que presenta elementos de la realidad histórica más inmediata y también de configuraciones narrativas asociados a la ficción.

Se sabe que los géneros, pese a estar condicionados históricamente, cuentan con elementos relativamente estables. ${ }^{43}$ La hagiografía, por su amplitud y flexibilidad, es adecuada para poner a prueba la perduración de ciertos rasgos que pueden aparecer en cualquier tiempo. La variabilidad de los personajes según el entorno o el modo en que se ven afectados por el paso del tiempo es uno de estos rasgos. Otro, en el que se centra esta este artículo, es la preponderancia relativa del polo sintagmático o del paradigmático, la importancia de la sucesión y la cronología o su relativa accesoriedad. En la hagiografía bizantina sobresale el polo paradigmático y la relación metafórica entre las partes de una obra. Esto puede deberse a que de manera transversal a los géneros ha surgido una nueva concepción de la perspectiva y de la unidad. En el período bizantino medio sus características principales ya están cristalizadas. Se trata de una unidad que, al contrario de la aristotélica, no es orgánica; la función de cada parte no está determinada por su relación con otras partes y con el todo sino por su relación con el exterior, con el mundo del auditorio que sí es orgánico debido a su entorno homogeneizador. Esta nueva unidad explica la discontinuidad de gran parte de la

43 Sobre lo "universal” en los géneros, véase JAUss (1982: 83). 
producción bizantina, la desproporcionalidad de las partes con el todo, o la tendencia a sumar episodios o reflexiones que no guardan relación con el tema principal si se las juzga con los criterios de unidad verificables en los autores clásicos. A su vez, esta nueva unidad le debe mucho a un libro que es en sí mismo genus [...] singulare, transcendens, nullam cum aliis comparationem ferens, a saber, la Biblia. Un enfoque tradicional ve esta influencia en el rol de la imitatio Christi de la hagiografía, que implica "algún tipo de relación entre las uitae y el Evangelio, puesto que éste es una biografía de Jesucristo" (GoNZÁlez Marín 2000: 315). No menos tradicional es entenderla al modo de VAN UyTFANGHE (1985: 572) desde la complementariedad: "Officiellement, la littérature relative à ces 'hommes' -et femmes- de Dieu [las passiones y las vitae] n'est pas devenue une 'Bible supplémentaire' [...] mais officieusement elle se présente comme une exégèse pratique de la Bible, comme une Bible 'actualisée"' o desde las huellas explícitas e implícitas (1985: 573): las primeras son "les références directes et les citations plus ou moins textuelles", las segundas "toute une série de procédés à base scripturaire" ${ }^{\prime 4}$.

44 En Van Uytfanghe (1985: 607-609) hay un apartado explícito sobre "Linfluence biblique sur la langue et le style des hagiographes." El autor encuentra "citations explicites, modèles implicites, langue et style" (1985: 609). El libro en el que se encuentra dicha contribución se detiene también en la influencia del latín bíblico sobre los autores de la antigüedad tardía y el Medioevo. Si bien sus observaciones están dirigidas ex-
El enfoque que interesa más en esta contribución es el que pone el acento en la estructura composicional. La Biblia, en efecto, derribó las barreras entre los géneros:

The abundance of literary forms and genres ascertainable in the Old and New Testaments is astonishing [...]. The Bible contains worldly lyrics (songs of work, ridicule, drinking, burial, and war) as well as spiritual ones (the hymn or the lament). It developed the most varied forms of narrative prose: etiological, historical, and also heroic sagas (the legendary garland for Samson); legends of martyrs and novellas (the King's novellas, but also the Book of Ruth). It contains the model for various forms of historiography (tribal legend, genealogy, royal chronicle), historical prose (documents, letters, contracts, war reports), and biography (the selfdisclosures of the prophets). All imaginable forms of wisdom literature (proverb, riddle, parable, fable, debate, allegory) and religious instruction (sermon, exhortation, epistle) are also found in $\mathrm{it}^{45}$.

Esta combinación de géneros, junto con la nueva concepción de unidad, favorece que numerosas obras cristianas combinen elementos que antes solían aparecer separados. Hemos visto la productividad del cronotopo mixto de la hagiografía, a la vez novelesco y biográfico. Queda

plícitamente a Occidente, salvo una breve discusión de la Vita Antonii, son pertinentes también para la hagiografía en lengua griega.

45 Jauss (1982: 102). 
por recordar que, así como la génesis de la biografía antigua es doble (historiográfica y encomiástica), sus métodos básicos dobles (el de la íđogí $\alpha$ y el del anticuarianismo), sus modos principales igualmente dobles (biografías peripatética y alejandrina, Plutarco y Suetonio) o, en una palabra, así como la biografía puede dividirse en un polo sintagmático y otro paradigmático, la hagiografía combina estos polos en una misma obra, dándole al paradigmático un peso tal que ni siquiera en un representante de la biografía alejandrina sería concebible. Las vilipendiadas categorías de LeO, ligeramente reescritas para convertirse en polos formales, pueden ser de utilidad para explicar obras de otros tiempos. Así parece haberlas entendido también BAJTín.

\section{Ediciones y traducciones}

Bartelink, G. J. M. (1994). Athanase d'Alexandrie. Vie d'Antoine. Paris: Cerf.

Büttner-Wobst, T. (1899-1905), Polybii historiae, vols. 1-4. Leipzig: Teubner.

Carras, L. (2017). "The Life of St Athanasia of Aegina: A critical edition with introduction", en A. Moffat (ed.), Maistor. Classical, Byzantine and Renaissance Studies for Robert Browning. Leiden - Boston: Brill ( $1^{\text {a }}$ ed. 1984); 199-224.

Cavallero, P. A. et al. (2009). Leoncio de Neápolis. Vida de Simeón el loco. Edición revisada con traducción, introducción y notas. Buenos Aires: Universidad de Buenos Aires.
Cavallero, P. A. et al. (2011). Leoncio de Neápolis. Vida de Juan el limosnero. Edición revisada con traducción, introducción, notas y apéndices. Buenos Aires: Universidad de Buenos Aires.

Dindorf, L. \& Thaleim, Th. (1915). Xenophontis Scripta minora. Agesilaus: 169-209. Leipzig: Teubner.

Fontaine, J. (1967). Sulpice Sévère. Tome I. Vie de Saint Martin. Introduction, texte et traduction. Paris: Cerf.

Hude, K. (1969). Xenophontis Historia Graeca (= Hellenica). Leipzig: Teubner (1 $1^{\text {a }}$ ed. 1934).

KASSEL, R. (1965). Aristotelis De arte poetica liber. Oxford: Oxford University Press.

Kaster, R.A. (2006). C. Suetoni Tranquilli De vita Caesarum libri VIII et De grammaticis et rhetoribus liber. Oxford: Oxford University Press.

Maraval, P. (1971). Grégoire de Nysse. Vie de Sainte Macrine. Paris: Cerf.

Migne, J.-P. 1863. Symeonis Logothetae cognomento Metaphrastae Opera omnia, III. Vita et conversatio S.P.N. Spyridonis, PG 116, 417-468. Parisiis: Imprimerie Catholique.

Verheijen, L. (1981). Sancturs Aurelius Augustinus. Confessiones, Turnhout: Brepols.

Ziegler, K. (1914). Plutarchi vitae parallelae. Leipzig: Teubner.

\section{Bibliografía citada}

Bakhtine, M. (1978). Esthétique et théorie du roman. Paris: Gallimard ( $1^{\text {a }} \mathrm{ed}$. rusa 1975).

Бахтин, М. М (2012). Собрание сочинений в 7-ми томах. Том 3. Теория романа (1930-1961 г2.). San Petersburgo: Институт мировой литературы. 
BeCK, H.-G. (1959). Kirche und theologische literatur im byzantinischen Reich. München: Beck.

Borges, J. L. (1974). Obras completas, 1923-1972. Buenos Aires: Emecé.

Curtius, E. R. (1948). Europäische Literatur und lateinisches Mittelalter. Bern/ München: Francke.

de Certeau, M. (1968): "Hagiographie" en Encyclopodia Universalis. Paris, Encyclopædia Universalis 8; 207-209.

Dihle, A. (1998). "Zur antiken Biographie" en EHLERs, W. W. La biographie antique (Entretiens sur l'Antiquité classique 44). Vanclouvres/ Genève: Fondation Hardt; 119-146.

Dilthey, W. (1965). Gesammelte Werke, vol. VII. Stuttgart: Vandenhoeck und Ruprecht.

DrüE, H. (1994). "Die Entwicklung des Begriffs Selbstgefühl in Philosophie und Psychologie": Archiv für Begriffsgeschichte 37; 285-305

DüRING, I. (1966). Aristoteles. Darstellung und Interpretation seines Denkens. Heidelberg: Winter.

ERLICH, V. (1980). Russian Formalism. History - Doctrine. The Hague: Mouton (1 $1^{\text {a }}$ ed. 1955).

Ferrer, E. (2013) "El género bíos desde la antigüedad hasta Gregorio de Nisa" en Alesso, M. (ed.), Hermenéutica de los géneros literarios: de la Antigüedad al Cristianismo. Buenos Aires: Universidad de Buenos Aires; 303-335.

Godman, P. (2019). From Poliziano to Machiavelli: Florentine Humanism in the High Renaissance. Princeton: Princeton University Press.

González Marín, S. (2000). Análisis de un género literario: las vidas de santo en la antigüedad tardía. Salamanca: Ediciones Universidad de Salamanca.
HäGG, Th. (2012). The art of Biography in Antiquity. Cambridge: Cambridge University Press.

Нirschкор, K. (1999). Mikhail Bakhtin. An Aesthetic for Democracy. Oxford/ New York: Oxford University Press.

Hirschкop, K. (2001). "Bakhtin in the sober light of day (introduction to the revised edition)" en Нirschкор, K. \& Shepherd, D. (eds.), Bakhtin and Cultural Theory. Manchester/ New York: Manchester University Press; 1-25.

JAUSs, H.R. (1982). "Theory of Genre and Medieval Literature”, en ID., Towards an Aesthetic of Reception. Minneapolis: University of Minnesota Press.

Konstan, D. \& Walsh, R. (2016). “Civic and subversive biography in Antiquity" en De Temmerman, K. \& DeMOEN, K. (eds.). Writing Biography in Greece and Rome: Narrative Technique and Fictionalization. Cambridge: Cambridge University Press; 26-43.

Lalanne, S. (2006). Une éducation grecque. Rites de passage et construction des genres dans le roman grec ancien. Paris: La Découverte.

LeO, F. (1901). Die griechisch-römische Biographie nach ihrer litterarischen Form. Leipzig: Teubner.

Misch, G. (1907). Geschichte der Autobiographie. Erster Band: das Altertum, Leipzig - Berlin: Teubner.

Momigliano, A. (1950). "Ancient History and the Antiquarian": Journal of the Warburg and Courtauld Institutes 13/3-4; 285-315.

Momigliano, A. (1993). The Development of Greek Biography. Cambridge (Mass.): Harvard University Press ( $1^{\mathrm{a}}$ ed. 1971).

Pratsch, Th. (2005). Der hagiographische Topos. Berlin/ New York: De Gruyter. 
Sonnabend, H. (2002). Geschichte der antiken Biographie. Von Isokrates bis zur Historia Augusta, Stuttgart: Metzler.

Stadter, Ph. A. (1991). "Fictional Narrative in the Cyropaideia": The American Journal of Philology 112/4 (1991); 461-491.

Van WaArden, J. (2020). "Amicitia, Otium, and the Chronotope of Sidonius's Correspondence": Journal of Late Antiquity 13/1 (2020); 149-172.

Van Uytfanghe, M. (1985). "L'empreinte biblique sur la plus ancienne hagiographie occidentale" en Fontaine, J. \& Pietri, C. Le monde latin antique et la Bible. Paris: Beauchesne; 565-613.

Van Uytfanghe, M. (2005). "La biographie classique et l'hagiographie chrétienne antique tardive": Hagiographica $12 ; 223-248$.

Wallace-Hadrill, A. (1995). Suetonius. London: Bristol Classical Press ( $1^{\mathrm{a}} \mathrm{ed}$. 1983).

West, S. (1974). "Satyrus: Peripatetic or Alexandrian"?: Greek, Roman and Byzantine Studies 15; 279-287.

Recibido: 16-09-2020

Evaluado: $11-10-2020$

Aceptado: 13-10-2020 